\title{
HARD THERMAL LOOPS AND CHIRAL LAGRANGIANS
}

\author{
Cristina Manuel \\ Dpt. Estructura i Constituents de la Matèria \\ Facultat de Física, Universitat de Barcelona \\ Diagonal 647, 08028 Barcelona (SPAIN)
}

\begin{abstract}
Chiral symmetry is used as the guiding principle to derive hard thermal loop effects in chiral perturbation theory. This is done by using a chiral invariant background field method for the non-linear sigma model and the WessZumino-Witten lagrangian, with and without external vector and axial vector sources. It is then shown that the $n$-point hard thermal loop is the leading thermal correction for the Green function of $n$ point vector soft quark currents.
\end{abstract}

PACS No: 12.39.Fe, 11.10.Wx, 11.30.Rd, 12.38.Bx.

ECM-UB-PF-97/27

October $/ 1997$ 


\section{INTRODUCTION}

At low energies QCD is successfully described in terms of effective chiral lagrangians 《1 4 . Those only involve the low energy modes or (pseudo) Goldstone bosons of the spontaneously broken chiral symmetry.

Chiral symmetry implies that at low energy the functional $\mathcal{Z}$ generating the connected Green's functions of vector and axial vector quark currents admits a representation of the form

$$
\exp (-i \mathcal{Z})=\int[d \Sigma] \exp \left(-i \int d x \mathcal{L}_{e f f}(\Sigma, v, a)\right)
$$

where $\Sigma$ is a $S U(N)$ unitary matrix, $N$ is the number of quark flavors, and $v_{\mu}$ and $a_{\mu}$ are external vector and axial vector sources, respectively. The effective lagrangian $\mathcal{L}_{\text {eff }}$ is expanded in powers of external momenta and of quark masses as

$$
\mathcal{L}_{\text {eff }}=\mathcal{L}_{2}+\mathcal{L}_{4}+\mathcal{L}_{6}+\ldots
$$

Lorentz invariance, and $P, C$ and chiral symmetry restricts the form of each term in the series. The matrix $\Sigma$ is parametrized in terms of pseudoscalar mesons $\pi$ as $\Sigma=\exp \left(i \pi / f_{\pi}\right)$, where $f_{\pi}$ can be identified, to first order, with the pion decay constant, $f_{\pi} \simeq 93 \mathrm{MeV}$.

At low but finite temperature $T$ chiral symmetry is still broken, and the use of effective chiral theory is still valid [5]. This is due to the fact that in the partition function the contribution of Goldstone states dominates over those of heavier particles, which are exponentially suppressed. The use of chiral effective theory is then justified for $T \ll f_{\pi}$, but as the $T$ is raised, the contribution of heavier states becomes important.

Symmetries which are spontaneously broken at zero temperature are usually restored at high $T[6]$. This is why it is expected that chiral symmetry is restored at enough high $T$. On the other hand, and due to asymptotic freedom, at very high T QCD is believed to be in an unconfined phase [7], the so called quark-gluon plasma phase. Therefore, there are two different phase transistions in QCD. It is yet an open question whether these occur together or not. Lattice computations seem to indicate that the critical temperature of the two phase transitions of QCD are of the same order [8].

At high $T$ and at one-loop a thermal mass for the gauge longitudinal degrees of freedom is generated, the so called Debye mass. To take into account properly the thermal effects of Debye screening a resummation of an infinite number of Feynman diagrams, the hard thermal loops (HTL's), is required [9]. Hard thermal loops are thermal amplitudes which arise in a gauge theory when the external loop momenta is soft, while the internal one is hard. Soft denotes a scale $\sim g T$, while hard refers to one $\sim T$, where $g \ll 1$ is the gauge coupling constant.

There has been a lot of studies on the HTL's of QCD (see Ref. [12] for a review). The effective action generating HTL's is a mass term for the chromoelectric fields, and it has been constructed just by solving a gauge invariance condition imposed on it [13]. This mass term is, however, non-local.

It has been recently claimed that HTL's also arise in the non-linear sigma model [10,11]. In a recent article Pisarski and Tytgat have shown that the same thermal amplitudes, the 
HTL's, for soft modes appear in that theory. In this context soft means a energy scale $\ll T$. In this chiral model HTL's do not describe a mass term, but just thermal corrections to $\pi-\pi$ scattering.

In this article I present an alternative derivation for the hard thermal effects in chiral perturbation theory. The main result of this paper is showing that chiral symmetry is the guiding principle which allows to extract the thermal effective action for soft modes in a chiral lagrangian. This is to be compared with the gauge symmetry principle which was imposed in a non-Abelian gauge theory to extract the HTL effective action.

The approach followed in this paper agrees in spirit with that of Ref. [10, but differs in some technical points. Pisarski and Tytgat used a background field method to extract the HTL's of a non-linear sigma model, and the Wess-Zumino-Witten lagrangian. However, their method was not manifestly chiral invariant. Here a chiral invariant background field method will be used instead to find the symmetry principle which is behind the HTL's of the theory. The thermal amplitudes obtained for momenta $P \ll T$ should agree on-shell for the two different background field methods [10]. However, using a manifestly invariant field expansion allows to express the one-loop corrections in terms of structures which have well defined properties under a chiral transformation. This is crucial when one has to construct higher order lagrangians in chiral perturbation theory.

This paper is structured as follows. In Sec. [1] a derivation of the HTL effective action in the non-linear sigma model is given. In Sec. III the results are generalized for the nonlinear sigma model with external vector and axial vector sources. The method of external sources allows to recognize that the $n$-point HTL, with $n=2, \ldots, \infty$, is the leading thermal correction for the Green function of $n$ soft vector quark currents. In Sec. IV the WessZumino-Witten terms, with and without external sources, are taken into consideration, and the hard thermal effects are studied. Once again, a symmetry principle allows to get the corresponding thermal effective action. Conclusions are presented in the last section. Throughout this article computations are done using the imaginary time formalism, and it is assumed that $T \ll f_{\pi}$.

\section{THE NON-LINEAR SIGMA MODEL}

Let us consider the lagrangian of the non-linear sigma model

$$
\mathcal{L}=\frac{f_{\pi}^{2}}{4} \operatorname{Tr}\left(\partial_{\mu} \Sigma^{\dagger} \partial_{\mu} \Sigma\right), \quad \Sigma^{\dagger} \Sigma=1
$$

where $\Sigma$ is a $S U(N)$ unitary matrix. The above lagrangian is invariant under a global $S U_{R}(N) \times S U_{L}(N)$ symmetry, where $\Sigma$ transforms as $\Sigma^{\prime}(x)=U_{R} \Sigma(x) U_{L}^{\dagger}$, and $U_{R, L} \in$ $S U_{R, L}(N)$.

A standard technique to evaluate the loop effects generated by the lagrangian $\mathcal{L}$ consists in expanding it around the solution of the classical equations of motion. The one-loop effective action is then obtained after integrating out the quantum fluctuations. There are several different ways to perform the expansion around the classical solution. In this section 
a background field expansion respectful with the symmetries of $\mathcal{L}$ will be performed to compute one-loop thermal effects.

Let $\bar{\Sigma}$ be the solution of the classical equations of motion derived from (2.1). One now defines the field $\xi(x)$ as the square root of $\bar{\Sigma}$ [3, 1 ]

$$
\bar{\Sigma}(x)=\xi(x) \xi(x) \text {. }
$$

The field $\Sigma$ is then written in the form

$$
\Sigma(x)=\xi(x) h(x) \xi(x) .
$$

It is interesting to study the transformation properties of these new variables. Under a global $S U_{R}(N) \times S U_{L}(N)$ transformation the field $\xi(x)$ behaves as

$$
\xi^{\prime}(x)=U_{R} \xi(x) U^{\dagger}(x)=U(x) \xi(x) U_{L}^{\dagger},
$$

while

$$
h^{\prime}(x)=U(x) h(x) U^{\dagger}(x)
$$

where $U$ is a unitary matrix which depends on $\xi(x)$ (and thus it depends on $x$ !), $U_{R}$ and $U_{L}^{\dagger}$. If $U_{R}=U_{L}$, then $U=U_{R}=U_{L}$ and then $U$ is associated to an ordinary $S U(N)$ transformation.

Let us write down the lagrangian (2.1) in terms of the new variables $\xi$ and $h$. It is not difficult to check

$$
\begin{aligned}
\mathcal{L} & =\frac{f_{\pi}^{2}}{4} \operatorname{Tr}\left(\partial_{\mu}(\xi h \xi)^{\dagger} \partial_{\mu}(\xi h \xi)\right) \\
& =\frac{f_{\pi}^{2}}{4} \operatorname{Tr}\left(D_{\mu} h^{\dagger} D_{\mu} h\right)
\end{aligned}
$$

where

$$
\begin{aligned}
D_{\mu} h & =\partial_{\mu} h+L_{\mu} h-h R_{\mu}, \\
D_{\mu} h^{\dagger} & =\partial_{\mu} h^{\dagger}-h^{\dagger} L_{\mu}+R_{\mu} h^{\dagger},
\end{aligned}
$$

with $L_{\mu}$ and $R_{\mu}$ being defined as

$$
L_{\mu}=\xi^{\dagger} \partial_{\mu} \xi, \quad R_{\mu}=\xi \partial_{\mu} \xi^{\dagger}
$$

One can deduce from (2.4) the transformation properties of the above fields under the global $S U_{R}(N) \times S U_{L}(N)$ symmetry. These read

$$
\begin{aligned}
R_{\mu}^{\prime}(x) & =U(x) R_{\mu}(x) U^{\dagger}(x)+U(x) \partial_{\mu} U^{\dagger}(x), \\
L_{\mu}^{\prime}(x) & =U(x) L_{\mu}(x) U^{\dagger}(x)+U(x) \partial_{\mu} U^{\dagger}(x) .
\end{aligned}
$$

At this point, one parametrizes $h=\exp \left(i \pi / f_{\pi}\right)$, where $\pi$ is an hermitian and traceless matrix. Then $\mathcal{L}$ is expanded keeping only terms up and including quadratic in $\pi$. The linear term in $\pi$ vanishes after imposing the classical equations of motion. One then gets 


$$
\mathcal{L}^{(0)}+\mathcal{L}^{(2)}=-f_{\pi}^{2} \operatorname{Tr}\left(A_{\mu}^{2}\right)+\frac{1}{4} \operatorname{Tr}\left(D_{\mu} \pi\right)^{2}-\frac{1}{4} \operatorname{Tr}\left(\left[A_{\mu}, \pi\right]\right)^{2},
$$

where

$$
\begin{gathered}
D_{\mu} \pi=\partial_{\mu} \pi+\left[V_{\mu}, \pi\right] \\
V_{\mu}(x)=\frac{L_{\mu}(x)+R_{\mu}(x)}{2}, \quad A_{\mu}(x)=\frac{L_{\mu}(x)-R_{\mu}(x)}{2} .
\end{gathered}
$$

It is possible to deduce from (2.5) and (2.10a)-(2.10b) how the fields $\pi, V_{\mu}$ and $A_{\mu}$ behave under a chiral transformation. Thus

$$
\begin{aligned}
\pi^{\prime}(x) & =U(x) \pi(x) U^{\dagger}(x), \\
V_{\mu}^{\prime}(x) & =U(x) V_{\mu}(x) U^{\dagger}(x)+U(x) \partial_{\mu} U^{\dagger}(x), \\
A_{\mu}^{\prime}(x) & =U(x) A_{\mu}(x) U^{\dagger}(x) .
\end{aligned}
$$

The field $V_{\mu}$ transforms like a connection, while $A_{\mu}$ and $\pi$ transforms covariantly. Thus, it can be immediately checked that under the above symmetry each term in (2.11) remains invariant. The lagrangian (2.11) looks formally as the one of a non-Abelian gauge theory, $V_{\mu}$ being the corresponding vector gauge field. This similarity is just formal, since there is not a kinetic term for $V_{\mu}$, neither for $A_{\mu}$, and thus those fields do not propagate.

The one-loop effective action of the non-linear sigma model is obtained by integrating out the $\pi$ fields. At zero temperature it can be done by evaluating the determinant of a differential operator, since the action is quadratic in the $\pi$ fields $[$ ?

It is our aim here to compute the leading thermal corrections to the effective action. The UV divergences which appear at finite $T$ are actually the same as the ones at $T=0$. The $T=0$ contributions to the effective action and the renormalization of the model will not be explicitly taken into account here. For the $T=0$ results see Ref. [3].

At this point it is interesting to make contact with the analysis of non-Abelian gauge theories at finite temperature. As pointed out by Pisarski and Tytgat, HTL's arise also in this model when the momenta of the background fields are soft, i.e. $\ll T$.

Let us first compute the thermal two-point functions $\left\langle V_{\mu}^{a} V_{\nu}^{b}\right\rangle_{T}$ and $\left\langle A_{\mu}^{a} A_{\nu}^{b}\right\rangle_{T}$. The computation of these functions is done here using the imaginary time formalism (ITF), following the techniques developed in Ref. [9].

In terms of the components $\pi^{a}$, we write $\pi=\pi^{a} \lambda^{a}$, where $\lambda^{a}$ is a generator of $S U(N)$ normalized as $\operatorname{Tr}\left(\lambda^{a} \lambda^{b}\right)=2 \delta^{a b}$, and $\left[\lambda^{a}, \lambda^{b}\right]=2 i f^{a b c} \lambda^{c}$, where $f^{a b c}$ are the structure constants of $S U(N)$. The $V_{\mu}$ two-point function then reads

$$
C_{V, \mu \nu}^{a b}(P)=f^{a d c} f^{b d c}\left(-2 \delta_{\mu \nu} \int \frac{d^{4} K}{(2 \pi)^{4}} \frac{1}{(K+P)^{2}}+\int \frac{d^{4} K}{(2 \pi)^{4}} \frac{(2 K+P)_{\mu}(2 K+P)_{\nu}}{K^{2}(K+P)^{2}}\right) .
$$

\footnotetext{
${ }^{1}$ Note also that the jacobian of the change of variables is unit at one-loop order.
} 
In ITF $K^{2}=k_{0}^{2}+\mathbf{k}^{2}$, and for bosonic fields $k_{0}=2 \pi n T$ for integral $n$. The standard notation

$$
\int \frac{d^{4} K}{(2 \pi)^{4}} \equiv T \sum_{n=-\infty}^{n=\infty} \int \frac{d^{3} k}{(2 \pi)^{3}}
$$

is used throughout this article.

The leading correction in $T$ when the external momentum $P$ is soft, i.e. $\ll T$, arises when the internal one $K$ is hard, i.e. $\sim T$. In this case, one can neglect $P$ in front of $K$ in the numerator of (2.15), and then one gets

$$
C_{V, \mu \nu}^{a b}(P) \approx-2 N \delta^{a b} \int \frac{d^{4} K}{(2 \pi)^{4}} \frac{\left(K^{2} \delta_{\mu \nu}-2 K_{\mu} K_{\nu}\right)}{K^{2}(K+P)^{2}} .
$$

The $S U(N)$ relation $f^{a d c} f^{b d c}=N \delta^{a b}$ has been used in Eq. (2.17).

It is possible to split the pure $T=0$ part from the finite $T$ contribution of the two-point function. We will only concentrate in the last one. Its dominant term can be extracted in the limit where the external momentum is soft, and it reads

$$
C_{V, \mu \nu}^{a b}(P, T) \approx-\delta^{a b} \frac{N T^{2}}{6} \delta \Pi_{\mu \nu}(P),
$$

where $\delta \Pi_{\mu \nu}(P)$ is the HTL arising in the polarization tensor of a gauge field theory [9]. This can be expressed as

$$
\delta \Pi_{\mu \nu}(P)=2 \delta_{\mu 0} \delta_{\nu 0}+2 i p_{0} \int \frac{d \Omega_{\mathbf{q}}}{4 \pi} \frac{Q_{\mu} Q_{\nu}}{Q \cdot P},
$$

where $Q=(i, \mathbf{q})$ is a null vector, $Q^{2}=0$, and the angular integral is over all directions of the three dimensional unit vector $\mathbf{q}$.

The thermal $A_{\mu}$ two-point function can be evaluated following a similar analysis. One then gets

$$
C_{A, \mu \nu}^{a b}(P, T) \approx \delta^{a b} \delta_{\mu \nu} \frac{N T^{2}}{6},
$$

and the $\approx$ sign means that only the leading thermal correction is retained.

Although the thermal correction in (2.20) gives a contribution to the effective action which is invariant under the symmetry (2.14a)-(2.14d), the thermal amplitude $\operatorname{Tr}\left(V_{\mu} \delta \Pi^{\mu \nu} V_{\nu}\right)$ does not. Since $V_{\mu}$ transforms as a connection under a chiral transformation it cannot appear in a chiral invariant effective action. However, as known from the studies of non-Abelian gauge theories, this is not the only thermal amplitude contributing to the effective action. There are also higher $n$-point functions, with $n$ arbitrary, which are relevant at the same order in the computation. Those can be determined by an explicit evaluation of each Feynman diagram [9].

In a gauge theory it has been proved that in the HTL approximation the quartic couplings of (2.11) only contribute in tadpole-like diagrams, but not in higher $n$-point functions. 
Therefore in the non-linear sigma model the only HTL involving the $A_{\mu}$ fields are the twopoint functions. This is also the reason why there are no mixed $A_{\mu}-V_{\mu}$ thermal amplitudes in this approximation. There is, however, an infinite set of HTL's involving the $V_{\mu}$ fields.

A symmetry argument allows to extract the complete thermal effective action for soft background fields, which is then expressed in terms of covariant objects. Following closely the work done in a gauge theory [15], one can write the effective action as

$$
\begin{aligned}
S+\delta S_{T} & =-f_{\pi}^{2}(T) \int d^{4} x \operatorname{Tr}\left(A_{\mu}^{2}(x)\right) \\
& -\frac{N T^{2}}{12} \int \frac{d \Omega_{\mathbf{q}}}{4 \pi} \int d^{4} x d^{4} y \operatorname{Tr}\left(V_{\mu \lambda}(x)<x\left|\frac{Q^{\mu} Q_{\nu}}{-(Q \cdot D)^{2}}\right| y>V^{\nu \lambda}(y)\right),
\end{aligned}
$$

where

$$
V_{\mu \nu}=\partial_{\mu} V_{\nu}-\partial_{\nu} V_{\mu}+\left[V_{\mu}, V_{\nu}\right] .
$$

Hard thermal effects change $f_{\pi}$ into $f_{\pi}(T)$ as

$$
f_{\pi}(T)=f_{\pi}\left(1-\frac{N}{24} \frac{T^{2}}{f_{\pi}^{2}}\right)
$$

which agrees with the result computed in the literature (see Ref. [0, 14]).

Notice that although the field strentgh $V_{\mu \nu}$ did not show up in $\mathcal{L}$, it is generated by the thermal corrections. In a real gauge field theory the second piece in (2.21), which is non-local, represents a gauge invariant mass term for the longitudinal degrees of freedom. In the non-linear sigma model, and after writing down $V_{\mu}$ in terms of the $\pi$ fields, the same HTL action represents thermal $\pi-\pi$ scattering [10] respectful with the symmetries of the theory.

In the static limit the non-local term in (2.21) becomes local and proportional to $\operatorname{Tr}\left(V_{0}^{2}\right)$. However, since in the static situation $V_{0}=A_{0}=0$, then the only hard thermal correction in (2.21) which survives in that case is $-f_{\pi}^{2}(T) \int d x \operatorname{Tr}\left(A_{i}^{2}\right)$.

At this point it is interesting to compare our results with the ones obtained by Pisarski and Tytgat. Those authors performed a different background field expansion to get the hard thermal effects. With some minor modifications in the notations, instead of the splitting (2.3), Pisarski and Tytgat defined $\Sigma(x)=\bar{\Sigma}(x) h(x), \bar{\Sigma}$ being the background field, and $h=\exp \left(i \pi / f_{\pi}\right), \pi$ being the quantum fluctuation. Written in terms of the $h$ fields, one then gets a lagrangian which looks formally the same as (2.6), but with $R_{\mu}=0$ and $\tilde{L}_{\mu}=\bar{\Sigma}^{\dagger} \partial_{\mu} \bar{\Sigma}$. After expanding $h=\exp \left(i \pi / f_{\pi}\right)$, and keeping only the terms up to quadratic in the $\pi$ fields, one then gets

$$
\mathcal{L}^{(0)}+\mathcal{L}^{(2)}=-\frac{f_{\pi}^{2}}{4} \operatorname{Tr}\left(\tilde{L}_{\mu}^{2}\right)+\frac{1}{4} \operatorname{Tr}\left(\tilde{D}_{\mu} \pi\right)^{2}-\frac{1}{16} \operatorname{Tr}\left(\left[\tilde{L}_{\mu}, \pi\right]\right)^{2},
$$

with $\tilde{D}_{\mu} \pi=\partial_{\mu} \pi+\frac{1}{2}\left[\tilde{L}_{\mu}, \pi\right]$. This lagrangian is not invariant under a standard $S U(N)$ transformation, since both the first and third terms break the symmetry. Nevertheless, Pisarski and Tytgat invoked a $S U(N)$ gauge symmetry principle to write the complete one-loop thermal effective action. According to Ref. [10], this was found to be 


$$
\begin{aligned}
\tilde{S}+\delta \tilde{S}_{T} & =-\frac{f_{\pi}^{2}(T)}{4} \int d^{4} x \operatorname{Tr}\left(\tilde{L}_{\mu}^{2}(x)\right) \\
& -\frac{N T^{2}}{12} \int \frac{d \Omega_{q}}{4 \pi} \int d^{4} x d^{4} y \operatorname{Tr}\left(\tilde{L}_{\mu \lambda}(x)<x\left|\frac{Q^{\mu} Q_{\nu}}{-(Q \cdot \tilde{D})^{2}}\right| y>\tilde{L}^{\nu \lambda}(y)\right),
\end{aligned}
$$

where

$$
\tilde{L}_{\mu \nu}=\partial_{\mu} \tilde{L}_{\nu}-\partial_{\nu} \tilde{L}_{\mu}+\frac{1}{2}\left[\tilde{L}_{\mu}, \tilde{L}_{\nu}\right] .
$$

Notice that the first term is not invariant under a gauge $S U(N)$ symmetry, while the second is by construction.

At first sight, Eqs. (2.21) and (2.25) seem to be different. However, they should give the same thermal amplitudes on-shell [10]. To check agreement between the two formalisms, use

of the Ward identities obeyed by the HTL's is required [16]. Here only agreement between the two-point functions will be checked in the lowest order in $1 / f_{\pi}$.

After writing $\xi=\exp \left(i \pi / 2 f_{\pi}\right)$, and expanding the exponentials, the $A_{\mu}$ and $V_{\mu}$ fields are expressed as

$$
A_{\mu}=\frac{i}{2 f_{\pi}} \partial_{\mu} \pi+O\left(\frac{1}{f_{\pi}^{3}}\right), \quad V_{\mu}=\frac{1}{8 f_{\pi}^{2}}\left[\pi, \partial_{\mu} \pi\right]+O\left(\frac{1}{f_{\pi}^{4}}\right) .
$$

Following Ref. [10] $\tilde{L}_{\mu}$ is given by

$$
\tilde{L}_{\mu}=\frac{i}{f_{\pi}} \partial_{\mu} \pi+\frac{1}{2 f_{\pi}^{2}}\left[\pi, \partial_{\mu} \pi\right]+O\left(\frac{1}{f_{\pi}^{3}}\right) .
$$

Since the HTL obeys the Ward identity $P_{\mu} \delta \Pi^{\mu \nu}(P)=0$, the contribution of the first term of (2.28) vanishes in the two-point function $\operatorname{Tr}\left(\tilde{L}_{\mu} \delta \Pi^{\mu \nu} \tilde{L}_{\nu}\right)$. I then found agreement between the two formalisms on-shell, except for a factor of 4 , which I think it was introduced incorrectly in (2.25) in Ref. [10].

\section{THE NON-LINEAR SIGMA MODEL WITH EXTERNAL SOURCES}

In this section we consider the non-linear sigma model

$$
\mathcal{L}_{2}=\frac{f_{\pi}^{2}}{4}\left(\operatorname{Tr}\left(\nabla_{\mu} \Sigma^{\dagger} \nabla_{\mu} \Sigma\right)+\operatorname{Tr}\left(\chi^{\dagger} \Sigma+\chi \Sigma^{\dagger}\right)\right), \quad \Sigma^{\dagger} \Sigma=1,
$$

where the covariant derivatives are defined as

$$
\nabla_{\mu} \Sigma=\partial_{\mu} \Sigma-i\left(v_{\mu}+a_{\mu}\right) \Sigma+i \Sigma\left(v_{\mu}-a_{\mu}\right),
$$

$v_{\mu}$ and $a_{\mu}$ being external vector and axial vector sources, respectively, and $\chi=B(s+i p)$, where $B$ is a constant, and $s$ and $p$ are scalar and pseudoscalar external sources, respectively.

It is convenient to introduce the combinations

$$
F_{\mu}^{R}=v_{\mu}+a_{\mu}, \quad F_{\mu}^{L}=v_{\mu}-a_{\mu} .
$$


Electromagnetic interactions in the non-linear sigma model can be considered as a special case of (3.1), just by setting $a_{\mu}=0$, and $v_{\mu}=F_{\mu}^{R}=F_{\mu}^{L}=Q A_{\mu}^{e . m}$, where $A_{\mu}^{e . m}$. is the electromagnetic gauge field, and $Q$ is the quark charge matrix, which for the $N=3$ case reads

$$
Q=\frac{e}{3}\left(\begin{array}{lll}
2 & & \\
& -1 & \\
& & -1
\end{array}\right) .
$$

Under the local $S U_{R}(N) \times S U_{L}(N)$ symmetry the fields transform as

$$
\begin{aligned}
\Sigma^{\prime}(x) & =U_{R}(x) \Sigma(x) U_{L}^{\dagger}(x), \\
F_{\mu}^{\prime R}(x) & =U_{R}(x) F_{\mu}^{R}(x) U_{R}^{\dagger}(x)+i U_{R}(x) \partial_{\mu} U_{R}^{\dagger}(x) \\
F_{\mu}^{\prime L}(x) & =U_{L}(x) F_{\mu}^{L}(x) U_{L}^{\dagger}(x)+i U_{L}(x) \partial_{\mu} U_{L}^{\dagger}(x), \\
\left(s^{\prime}(x)+i p^{\prime}(x)\right) & =U_{R}(x)(s(x)+i p(x)) U_{L}^{\dagger}(x) .
\end{aligned}
$$

The same analysis as in the previous section will be followed to compute the leading thermal corrections of the one-loop effective action, working in the exact chiral limit, so that $s=p=0$. In this limit the classical equations of motion derived from (3.1) are

$$
\bar{\Sigma} \nabla_{\mu} \nabla_{\mu} \bar{\Sigma}^{\dagger}-\nabla_{\mu} \nabla_{\mu} \bar{\Sigma} \bar{\Sigma}^{\dagger}=0
$$

As in Sec. II, one then defines

$$
\Sigma(x)=\xi(x) h(x) \xi(x)
$$

where $\bar{\Sigma}=\xi^{2}$ is the classical solution to the equations of motion. The transformation properties of $\xi$ and $h$ under the local $S U_{R}(N) \times S U_{L}(N)$ symmetry are

$$
\begin{aligned}
\xi^{\prime}(x) & =U_{R}(x) \xi(x) U^{\dagger}(x)=U(x) \xi(x) U_{L}^{\dagger}(x), \\
h^{\prime}(x) & =U(x) h(x) U^{\dagger}(x),
\end{aligned}
$$

where $U$ is a unitary matrix which depends on $\xi(x), U_{R}(x)$ and $U_{L}^{\dagger}(x)$.

Then one writes $h=\exp \left(i \pi / f_{\pi}\right)$ and expands the exponentials, keeping only terms up and including the quadratic in $\pi$ in the lagrangian. Thus

$$
\mathcal{L}_{2}^{(0)}+\mathcal{L}_{2}^{(2)}=\mathcal{L}_{2}(\bar{\Sigma})+\frac{1}{4} \operatorname{Tr}\left(\nabla_{\mu}\left(\xi^{\dagger} \pi \xi^{\dagger}\right) \nabla_{\mu}(\xi \pi \xi)-\frac{1}{2} \nabla_{\mu} \bar{\Sigma}^{\dagger} \nabla_{\mu}\left(\xi \pi^{2} \xi\right)-\frac{1}{2} \nabla_{\mu} \bar{\Sigma} \nabla_{\mu}\left(\xi^{\dagger} \pi^{2} \xi^{\dagger}\right)\right) .
$$

It is possible to express the above lagrangian as [3]

$$
\mathcal{L}_{2}^{(0)}+\mathcal{L}_{2}^{(2)}=-f_{\pi}^{2} \operatorname{Tr}\left(\Delta_{\mu}\right)^{2}+\frac{1}{4} \operatorname{Tr}\left(d_{\mu} \pi\right)^{2}-\frac{1}{4} \operatorname{Tr}\left(\left[\Delta_{\mu}, \pi\right]\right)^{2},
$$

where 


$$
\begin{aligned}
d_{\mu} \pi & =\partial_{\mu} \pi+\left[\Gamma_{\mu}, \pi\right], \\
\Gamma_{\mu} & =\frac{1}{2}\left(\xi^{\dagger} \nabla_{\mu}^{R} \xi+\xi \nabla_{\mu}^{L} \xi^{\dagger}\right), \\
\Delta_{\mu} & =\frac{1}{2}\left(\xi^{\dagger} \nabla_{\mu}^{R} \xi-\xi \nabla_{\mu}^{L} \xi^{\dagger}\right), \\
\nabla_{\mu}^{l} & =\partial_{\mu}-i F_{\mu}^{l}, \quad l=R, L .
\end{aligned}
$$

The transformation rules obeyed by the new fields are then deduced from (3.8a) - (3.8b)

$$
\begin{aligned}
\pi^{\prime}(x) & =U(x) \pi(x) U^{\dagger}(x), \\
\Gamma_{\mu}^{\prime}(x) & =U(x) \Gamma_{\mu}(x) U^{\dagger}(x)+U(x) \partial_{\mu} U^{\dagger}(x), \\
\Delta_{\mu}^{\prime}(x) & =U(x) \Delta_{\mu}(x) U^{\dagger}(x) .
\end{aligned}
$$

The lagrangian (3.10) looks like the same as the one in (2.11), with $\Gamma_{\mu}$ and $\Delta_{\mu}$ playing the same role as the $V_{\mu}$ and $A_{\mu}$ fields, respectively.

The same analysis as the one performed in the previous section leads to the thermal one-loop effective action for soft background fields $\Gamma_{\mu}$ and $\Delta_{\mu}$. It reads

$$
\begin{aligned}
\mathcal{Z}_{2}=S_{2}+\delta S_{2, T} & =-f_{\pi}^{2}(T) \int d^{4} x \operatorname{Tr}\left(\Delta_{\mu}^{2}(x)\right) \\
& -\frac{N T^{2}}{12} \int \frac{d \Omega_{\mathbf{q}}}{4 \pi} \int d^{4} x d^{4} y \operatorname{Tr}\left(\Gamma_{\mu \lambda}(x)<x\left|\frac{Q^{\mu} Q_{\nu}}{-(Q \cdot d)^{2}}\right| y>\Gamma^{\nu \lambda}(y)\right)
\end{aligned}
$$

where

$$
\Gamma_{\mu \nu}=\partial_{\mu} \Gamma_{\nu}-\partial_{\nu} \Gamma_{\mu}+\left[\Gamma_{\mu}, \Gamma_{\nu}\right]
$$

For later convenience, an identity obeyed by $\Gamma_{\mu \nu}$ is given [3]

$$
\Gamma_{\mu \nu}=-\left[\Delta_{\mu}, \Delta_{\nu}\right]-\frac{1}{2} F_{\mu \nu}^{+}
$$

where

$$
F_{\mu \nu}^{ \pm}=i\left(\xi F_{\mu \nu}^{L} \xi^{\dagger} \pm \xi^{\dagger} F_{\mu \nu}^{R} \xi\right)
$$

Chiral symmetry implies that the Green's functions associated with the vector and axial vector quark currents

$$
J_{\mu}^{a}(x)=\bar{q}(x) \frac{\lambda^{a}}{2} \gamma_{\mu} q(x), \quad J_{\mu 5}^{a}(x)=\bar{q}(x) \frac{\lambda^{a}}{2} \gamma_{\mu} \gamma_{5} q(x)
$$

can be obtained in the lowest order in the external momenta from the generating functional $\mathcal{Z}_{2}$. This can be done by performing functional derivatives of $\mathcal{Z}_{2}$ with respect to the external sources. Thus

$$
\begin{aligned}
& \left.\frac{\delta^{2} \mathcal{Z}_{2}}{\delta a_{\mu}^{a}(x) \delta a_{\nu}^{b}(y)}\right|_{a=v=0}=\left\langle J_{\mu 5}^{a}(x) J_{\nu 5}^{b}(y)\right\rangle \\
& \left.\frac{\delta^{2} \mathcal{Z}_{2}}{\delta v_{\mu}^{a}(x) \delta v_{\nu}^{b}(y)}\right|_{a=v=0}=\left\langle J_{\mu}^{a}(x) J_{\nu}^{b}(y)\right\rangle .
\end{aligned}
$$


Thermal effects modify the above two Greens functions with respect to their $T=0$ values [5,14]. Furthermore, the HTL effective action gives account of the leading thermal $n$-point vector quark current amplitudes. In momentum space, and for $P_{i} \ll T$, one has

$$
\left\langle J_{\mu_{1}}^{a_{1}}\left(P_{1}\right) \ldots J_{\mu_{n}}^{a_{n}}\left(P_{n}\right)\right\rangle_{T} \approx(-i)^{n} \frac{N T^{2}}{12} \delta \Gamma_{\mu_{1} \ldots \mu_{n}}^{a_{1} \ldots a_{n}}\left(P_{1}, \ldots, P_{n}\right)+O\left(\frac{1}{f_{\pi}^{2}}\right),
$$

where $\delta \Gamma_{\mu_{1} \ldots \mu_{n}}^{a_{1} \ldots a_{n}}\left(P_{1}, \ldots, P_{n}\right)$ is the $n$-point HTL.

\section{THE WESS-ZUMINO-WITTEN LAGRANGIAN}

To incorporate correctly the anomalies of QCD in presence of external vector and axial vector fields one has also to include in the chiral lagrangian the Wess-Zumino terms [17]. In chiral perturbation theory those terms are of order $P^{4}$. In this section the leading thermal corrections generated by those new terms will be computed. Computations at finite $T$ with the Wess-Zumino-Witten (WZW) lagrangian have already been considered in Ref. [19, 10,11].

The Wess-Zumino-Witten action in the absence of external sources can be expressed as an integral in five dimensions as [17]

$$
S_{W Z}(\Sigma)=-\frac{i N_{c}}{240 \pi^{2}} \int_{\partial D=M^{4}} \epsilon^{\alpha \beta \gamma \delta \rho} \operatorname{Tr}\left(\Sigma^{-1} \partial_{\alpha} \Sigma \Sigma^{-1} \partial_{\beta} \Sigma \Sigma^{-1} \partial_{\gamma} \Sigma \Sigma^{-1} \partial_{\delta} \Sigma \Sigma^{-1} \partial_{\rho} \Sigma\right) d^{5} x .
$$

In Eq. (4.1) $N_{c}$ is the number of colors, and the integral is over a five dimensional surface $D$ whose boundary is the four dimensional space.

In the presence of external vector and axial vector gauge fields the Wess-Zumino-Witten action is given by [18]

$$
\begin{aligned}
S_{W Z}\left(F^{L}, F^{R}, \Sigma\right) & =-\frac{i N_{c}}{240 \pi^{2}} \int_{\partial D=M^{4}} \operatorname{Tr}\left(\Sigma^{-1} d \Sigma\right)^{5} \\
& -\frac{N_{c}}{48 \pi^{2}} \int \operatorname{Tr}\left(-i F^{R}\left(d \Sigma \Sigma^{-1}\right)^{3}-P . C .\right. \\
& +\left(-F^{R} d F^{R}-d F^{R} F^{R}+i\left(F^{R}\right)^{3}\right)\left(-i \Sigma F^{L} \Sigma^{-1}+\Sigma^{-1} d \Sigma\right)-P . C . \\
& -\left(d \Sigma \Sigma^{-1} d F^{R} \Sigma F^{L} \Sigma^{-1}-P . C .\right) \\
& -\frac{1}{2}\left(F^{R} d \Sigma \Sigma^{-1} F^{R} d \Sigma \Sigma^{-1}-P . C .\right) \\
& -\left(\Sigma F^{L} \Sigma^{-1} F^{R} d \Sigma \Sigma^{-1} d \Sigma \Sigma^{-1}-P . C .\right) \\
& -i\left(F^{L} \Sigma^{-1} d \Sigma F^{L} \Sigma^{-1} F^{R} \Sigma-P . C .\right) \\
& \left.-\frac{1}{2}\left(F^{L} \Sigma^{-1} F^{R} \Sigma F^{L} \Sigma^{-1} F^{R} \Sigma\right)\right)
\end{aligned}
$$

where P.C. stands for parity conjugate: $F^{L} \leftrightarrow F^{R}, \Sigma \leftrightarrow \Sigma^{-1}$. The language of differential forms has been used in (4.2), so $d \Sigma=\partial_{\mu} \Sigma d x^{\mu}, F^{L, R}=F_{\mu}^{L, R} d x^{\mu}$, etc.

A background field expansion of the total lagrangian $\mathcal{L}_{2}+\mathcal{L}_{W Z}$ will be performed keeping only the quadratic terms in the fluctuations. In order to carry out this expansion it is very 
convenient to use the techniques advocated by Akhoury and Alfakih [20]. Those consist in defining a curve on the group manifold which passes through both the background field $\bar{\Sigma}$ and the background field with the fluctuation $\Sigma$. One then introduces a one-parameter family of group elements $\Sigma(x, t)$ such that

$$
\Sigma(x, 0)=\bar{\Sigma}(x)=\xi^{2}(x), \quad \Sigma(x, 1)=\Sigma(x)=\xi(x) \exp \left(i \pi / f_{\pi}\right) \xi(x) .
$$

A $t$-dependent action $S(t)$ is then defined. The action incorporating the fluctuations to all orders can be written as

$$
S(1)-S(0)=\int_{0}^{1} d t \frac{\partial S(t)}{\partial t}
$$

By introducing an exponential parametrization of the group element $\Sigma(x, t)=$ $\xi \exp \left(i t \pi / f_{\pi}\right) \xi$, the r.h.s. of (4.4) can be straightforwardly computed. It is enough for our purposes to keep only the quadratic term in $\pi$, which can be expressed as an integral in four dimensions. Details of this computation, as well as $T=0$ one-loop corrections to the WZW action can be found in Ref. [20].

The quadratic term in $\pi$ coming from the expansion of $\mathcal{L}_{2}$ has already been computed in Sec. III. The quadratic term in the fluctuation arising from $\mathcal{L}_{W Z}$ is [20]

$$
\begin{aligned}
\mathcal{L}_{W Z}^{(2)} & =-\frac{i N_{c}}{48 \pi^{2} f_{\pi}^{2}} \epsilon^{\mu \nu \alpha \beta} \operatorname{Tr}\left(\left(\pi d_{\mu} \pi-d_{\mu} \pi \pi\right)\left\{\Gamma_{\nu \alpha}, \Delta_{\beta}\right\}\right. \\
& +\left(\pi \Delta_{\mu} d_{\nu} \pi-d_{\nu} \pi \Delta_{\mu} \pi\right)\left(\Gamma_{\alpha \beta}-2 \Delta_{\alpha} \Delta_{\beta}\right) \\
& \left.-\frac{1}{8} \pi^{2}\left[\Gamma_{\mu \nu}-2 \Delta_{\mu} \Delta_{\nu}, F_{\alpha \beta}^{-}\right]-\frac{1}{2} \pi \Delta_{\mu} \pi\left\{F_{\nu \alpha}^{-}, \Delta_{\beta}\right\}\right),
\end{aligned}
$$

where the covariant derivative $d_{\mu}$ and the fields $\Gamma_{\mu \nu}, \Delta_{\mu}$ and $F_{\mu \nu}^{-}$have already been defined in Sec. III.

It is possible to express the complete quadratic term as

$$
\mathcal{L}_{2}^{(2)}+\mathcal{L}_{W Z}^{(2)}=\frac{1}{2}\left(\left(\partial_{\mu} \pi^{a}+\left(\Gamma_{\mu}^{a b}+S_{\mu}^{a b}\right) \pi^{b}\right)^{2}-\pi^{a}\left(\sigma_{0}^{a b}+\sigma_{W Z}^{a b}\right) \pi^{b}+\pi^{a} S_{\mu}^{a c} S_{\mu}^{c b} \pi^{b}\right),
$$

where

$$
\begin{aligned}
\Gamma_{\mu}^{a b}= & -\frac{1}{2} \operatorname{Tr}\left(\left[\lambda^{a}, \lambda^{b}\right] \Gamma_{\mu}\right) \\
\sigma_{0}^{a b}= & \frac{1}{2} \operatorname{Tr}\left(\left[\lambda^{a}, \Delta_{\mu}\right]\left[\lambda^{b}, \Delta_{\mu}\right]\right) \\
S^{\mu a b}= & \frac{i N_{c}}{48 \pi^{2} f_{\pi}^{2}} \epsilon^{\mu \nu \alpha \beta} \operatorname{Tr}\left(\left[\lambda^{a}, \lambda^{b}\right]\left\{\Gamma_{\nu \alpha}, \Delta_{\beta}\right\}-\left(\lambda^{a} \Delta_{\nu} \lambda^{b}-\lambda^{b} \Delta_{\nu} \lambda^{a}\right)\left(\Gamma_{\alpha \beta}-2 \Delta_{\alpha} \Delta_{\beta}\right)\right) \\
\sigma_{W Z}^{a b}= & \frac{i N_{c}}{96 \pi^{2} f_{\pi}^{2}} \epsilon^{\mu \nu \alpha \beta} \operatorname{Tr}\left(\frac{1}{4}\left\{\lambda^{a}, \lambda^{b}\right\}\left[F_{\mu \nu}^{-}, \Gamma_{\alpha \beta}-2 \Delta_{\alpha} \Delta_{\beta}\right]\right. \\
& \left.-\left(\lambda^{a} \Delta_{\mu} \lambda^{b}+\lambda^{b} \Delta_{\mu} \lambda^{a}\right)\left\{\Delta_{\nu}, F_{\alpha \beta}^{-}\right\}\right) .
\end{aligned}
$$

Notice that both $S_{\mu}$ and $\sigma_{W Z}$ transform covariantly under a chiral transformation. It should also be remarked that there is not a quadratic term in $S_{\mu}$ in (4.6). This is natural, since all the corrections coming from the Wess-Zumino lagrangian are proportional to $\epsilon^{\mu \nu \alpha \beta}$. 
In the special case where the external sources vanish $F_{\mu}^{L}=F_{\mu}^{R}=0$, then $\Gamma_{\mu}=V_{\mu}$ and $\Delta_{\mu}=A_{\mu}$. In this case $\sigma_{W Z}=0$, and using the identity (3.15) for vanishing external sources one can express

$$
S^{\mu a b}=-\frac{i N_{c}}{12 \pi^{2} f_{\pi}^{2}} \epsilon^{\mu \nu \alpha \beta} \operatorname{Tr}\left(\left[\lambda^{a}, \lambda^{b}\right] A_{\nu} A_{\alpha} A_{\beta}-\left(\lambda^{a} A_{\nu} \lambda^{b}-\lambda^{b} A_{\nu} \lambda^{a}\right) A_{\alpha} A_{\beta}\right) .
$$

We will only compute thermal corrections for soft background fields when there is only one anomalous vertex and one or several non-anomalous ones. Thermal corrections with several anomalous vertices are suppressed in chiral perturbation theory.

Thermal amplitudes involving only one vertex $\sigma_{W Z}$ do not produce any one-loop correction in the hard thermal approximation. The only Feynman diagram that in principle could give a hard thermal correction is a tadpole-like diagram proportional to $\sigma_{W Z}^{a a}$, where the $S U(N)$ index is summed. However, it can be checked that $\sigma_{W Z}^{a a}=0$. This is the reason why the coefficient of the Wess-Zumino lagrangian does not receive one-loop corrections, both at $T=0$ or at finite $T$. This result could be expected since that coefficient is fixed by topology [17.

Let us now evaluate a two-point function with one anomalous vertex $S_{\mu}$ and one nonanomalous one $\Gamma_{\mu}$. Due to the particular form of the lagrangian one gets the same momentum dependence than in (2.15). Therefore, in the situation where the external momenta is soft, the same leading thermal correction is extracted. Thus

$$
\frac{T^{2}}{12} \int \frac{d^{4} P}{(2 \pi)^{4}} S_{\mu}^{a b}(P) \delta \Pi^{\mu \nu}(P) \Gamma_{\nu}^{a b}(-P)
$$

There are also other amplitudes with one anomalous vertex $S_{\mu}$ and $n$ non-anomalous ones $\Gamma_{\mu}$ which give a non-trivial contribution to the thermal effective action. Instead of analyzing those Feynman diagrams a symmetry argument will be used to obtain the complete thermal effective action, as it was done in the previous sections.

By going from momentum to coordinate space, Eq. (4.9) can be expressed as

$$
\frac{T^{2}}{12} \int d^{4} x d^{4} y \int \frac{d \Omega_{q}}{4 \pi}\left(\partial_{\mu} \Gamma_{\alpha}^{a b}-\partial_{\alpha} \Gamma_{\mu}^{a b}\right)<x\left|\frac{Q^{\alpha} Q^{\beta}}{-(Q \cdot \partial)^{2}}\right| y>\left(\partial_{\mu} S_{\beta}^{a b}-\partial_{\beta} S_{\mu}^{a b}\right) .
$$

Using the $S U(N)$ relation

$$
\sum_{a=1}^{N^{2}-1} \operatorname{Tr}\left(\lambda^{a} A\right) \operatorname{Tr}\left(\lambda^{a} B\right)=2 \operatorname{Tr}(A B)-\frac{2}{N} \operatorname{Tr} A \operatorname{Tr} B,
$$

Eq. (4.10) becomes

$$
\frac{T^{2}}{12} \int d^{4} x d^{4} y \int \frac{d \Omega_{q}}{4 \pi} \operatorname{Tr}\left(\left(\partial_{\mu} \Gamma_{\alpha}-\partial_{\alpha} \Gamma_{\mu}\right)<x\left|\frac{Q^{\alpha} Q^{\beta}}{-(Q \cdot \partial)^{2}}\right| y>\left(\partial_{\mu} S_{\beta}-\partial_{\beta} S_{\mu}\right)\right),
$$

where $S_{\mu}$ is defined by

$$
\begin{aligned}
S^{\mu}= & -\frac{i N_{c}}{48 \pi^{2} f_{\pi}^{2}} \epsilon^{\mu \nu \alpha \beta} \sum_{a=1}^{N^{2}-1}\left(\left[\left[\lambda^{a},\left\{\Gamma_{\nu \alpha}, \Delta_{\beta}\right\}\right], \lambda^{a}\right]\right. \\
& \left.-\left[\Delta_{\nu} \lambda^{a}\left(\Gamma_{\alpha \beta}-2 \Delta_{\alpha} \Delta_{\beta}\right)-\left(\Gamma_{\alpha \beta}-2 \Delta_{\alpha} \Delta_{\beta}\right) \lambda^{a} \Delta_{\nu}, \lambda^{a}\right]\right) .
\end{aligned}
$$


To make the whole expression (4.12) invariant under a chiral transformation it is enough to substitute the ordinary derivatives $\partial_{\mu}$ by covariant ones, $d_{\mu}$. Then one gets

$$
\frac{T^{2}}{12} \int d^{4} x d^{4} y \int \frac{d \Omega_{q}}{4 \pi} \operatorname{Tr}\left(\Gamma_{\mu \alpha}<x\left|\frac{Q^{\alpha} Q^{\beta}}{-(Q \cdot d)^{2}}\right| y>\left(d_{\mu} S_{\beta}-d_{\beta} S_{\mu}\right)\right)
$$

which gives the thermal effective action for soft background fields involving only one anomalous vertex.

From this effective action one could get the thermal corrections to several anomalous decays, such as $\pi^{0} \rightarrow \gamma \gamma$ [1].

\section{CONCLUSIONS}

In a non-Abelian gauge theory the one-loop thermal correction to the polarization tensor is easily computed when the external momenta of the gluon is soft, $P \sim g T \ll T$. Recquiring these thermal effects be gauge invariant allows to obtain the one-loop thermal effective action for soft modes. This effective action describes thermal amplitudes with $n$ external soft gluon legs, with $n=2, \ldots, \infty$, the so called HTL's, and represents a thermal mass for the longitudinal gauge degrees of freedom.

In the framework of chiral perturbation theory also a symmetry argument allows to derive the complete hard thermal loop effects from the knowledge of the two-point functions. This is the idea that has been exploited in this article.

The same thermal amplitudes, the HTL's, appear in a gauge field theory and in a chiral lagrangian. However, it should be clear that the physics associated to the HTL's is quite different in the two different models, as it has been already emphasized.

In a gauge theory HTL's are thermal amplitudes which are as important as the tree amplitudes for soft momenta, and thus they have to be resummed into effective vertices and propagators. The gauge coupling constant $g$, which at high $T$ is $g \ll 1$, allows to identify neatly the two different energy scales $T$ and $g T$ as two relevant physical scales of the theory.

In the chiral lagrangians that have been considered here HTL's only represent corrections to $\pi-\pi$ scattering of order $T^{2} / f_{\pi}^{2}$, and they do not have to be resummed. It is actually the dimensionless quantity $T^{2} / f_{\pi}^{2}$, which is assumed to be $T^{2} / f_{\pi}^{2} \ll 1$, the expansion parameter for the thermal corrections in chiral perturbation theory. It should be stressed that this pa-

rameter allows also to separate neatly the physical scales $\operatorname{hard}(\sim T)$ or $\operatorname{soft}\left(\sim \sqrt{\left(T^{2} / f_{\pi}^{2}\right)} T\right)$ in a chiral theory.

A power counting analysis could be established to obtain the leading thermal corrections in an arbitrary Feynman diagram in chiral perturbation theory. This counting is essential to get correctly the two-loop thermal corrections arising from $\mathcal{L}_{2}$, and one-loop thermal corrections from $\mathcal{L}_{4}$. It is then a remaining open question whether one could study those thermal effects with the same kind of techniques that have been used in this article. 


\section{Acknowledgments:}

I want to thank F. Brandt, V. Koulovassilopoulos and J. Taron for useful discussions, and to R. Jackiw for pointing out the relevance of some references. This work has been supported by funds provided by the CICYT contract AEN95-0590, and by the CIRIT contact GRQ931047. 


\section{REFERENCES}

[1] S. Weinberg, Physica 96 A (1979) 327.

[2] S. Coleman, J. Wess, and B. Zumino, Phys. Rev. 117 (1969); C. G. Callan, S. Coleman, J. Wess, and B. Zumino, ibid 117 (1969) 2247.

[3] J. Gasser and H. Leutwyler, Ann. Phys. 158 (1984) 142; J. Gasser and H. Leutwyler, Nucl. Phys. B250 (1985) 465.

[4] For a general review on chiral perturbation theory see, e.g. J. F. Donoghue, E. Golowich, and B. R. Holstein, "Dynamics of the Standard Model', Cambridge University Press, New York 1996; H. Georgi, "Weak Interactions and Modern Particle Theory", Benjamin/Cummings (1984).

[5] J. Gasser and H. Leutwyler, Phys. Lett. B184 (1987) 83.

[6] L. Dolan and R. Jackiw, Phys. Rev. D9 (1974) 3320; S. Weinberg, Phys. Rev. D9 (1974) 3357.

[7] J. C. Collins and M. J. Perry, Phys. Rev. Lett. 34 (1975) 1353; N. Cabibbo and G. Parisi, Phys. Lett. B59 (1975) 67.

[8] H. Meyer-Ortmanns, Rev. Mod. Phys. 68 (1996) 473.

[9] R. D. Pisarski, Phys. Rev. Lett. 63 (1989) 1129; E. Braaten and R. Pisarski, Nucl. Phys. B337 (1990) 569; J. Frenkel and J. C. Taylor, Nucl. Phys. B334 (1990) 199.

[10] R. D. Pisarski and M. Tytgat, Phys. Rev. Lett. 78 (1997) 3622.

[11] R. D. Pisarski, T. L. Trueman, and M. Tytgat, hep-ph/9702362, to be published in Phys. Rev. D

[12] M. Le Bellac, "Thermal Field Theory”, Cambridge University Press, Cambridge 1996.

[13] J. C. Taylor and S. M. H. Wong, Nucl. Phys. B346 (1990) 115.

[14] P. Binetruy and M. K. Gaillard, Phys. Rev. D32 (1985) 931; M. Dey, V. L. Eletsky, and B. L. Ioffe, Phys. Lett. B252 (1990) 620; A. Bochkarev and J. Kapusta, Phys. Rev. D54 (1996) 4006; R. D. Pisarski and M. Tytgat, ibid D54 (1996) 2989.

[15] E. Braaten and R. Pisarski, Phys. Rev. D45 (1992) R1827.

[16] E. Braaten and R. Pisarski, Nucl. Phys. B339 (1990) 310.

[17] J. Wess and B. Zumino, Phys. Lett. B37 (1971) 95; E. Witten, Nucl. Phys. B223 (1983) 422.

[18] L. Alvarez-Gaumé and P. Ginsparg, Ann. Phys. 161 (1985) 423.

[19] R. F. Alvarez-Estrada, A. Dobado and A. Gomez-Nicola, Phys. Lett. B319 (1993) 238; Phys. Lett. B324 (1994) 345. 
[20] R. Akhoury and A. Alfakih, Ann. Phys. 210 (1991) 81. 